Acta vet. scand. $1967,8,186-188$

Brief Communication

\title{
MYCOPLASMOSIS: INFECTIONS OF THE GENITAL ORGANS OF BULLS
}

In Nov. 1966 mycoplasmas were cultured from a pus-filled semen sample of a one-year-old A.I. bull " $K$ ". In subsequent samples pus admixture continued, and mycoplasmas were isolated also in Dec. When the pathogenicity of the strain " $K$ " had been proved, the bull " $K$ ", 14 months old, was purchased for further examination, and two normal twin bulls, 19 months old, were experimentally infected Jan. 10, 1967.

Methods: Experiment 1. Bull no. 89 was infected in both vesicular glands by inoculation of $2 \mathrm{ml} \times 3$ of a 72-hour-old broth culture, through the rectal wall, a technique first used by Christensen (1948), later by Blom \& Dam (1967). Number of viable cells per ml was 105 .

Experiment 2. Bull no. 90 was infected in the left testis, proximally and distally, by inoculation of $3 \mathrm{ml} \times 2$ of the culture used in experiment 1.

Clinical and laboratory findings. Experiment 1. On the third day the right vesicular gland was enlarged to about double size, while the left one was not enlarged until the 14th day, at which time both glands were firm and hard. Gray-brown semen with clots of pus was collected on the third day. Pus admixture continued till slaughtering on the 23rd day. Mycoplasmas were isolated from the third day. Post mortem: All genital organs appeared normal except the vesicular glands, of which the left one was twice as big as normal, distinctly firm. The cut surface yielded a creamy, yellow secretion. The right gland, about $50 \%$ enlarged, had haemorrhagic and pus-filled foci subcapsularly; the cut surface was dry, brownish, with a sparce secretion, only present as honey-like drops. Microscopic examination revealed pathological changes of the vesicular glands only. The lesions were similar to those found in the mammary gland (Ern $\phi 1967$ ), i.e., a progressive, eosinophilic inflammation showing an acute phase with extensive accumulations of eosinophilic granulocytes 
in the interstitia and alveoli, and a chronic phase with epithelial and lymphoid hyperplasia, plasma cell infiltration and fibrosis. Mycoplasmas could be recovered from both vesicular glands, ampullae and the prepuce.

Experiment 2. After one month the left ampulla was firm and slightly enlarged. Two weeks later a hard swelling appeared, of hazel-nut size, in the caput epididymidis. Semen samples were normal until the 22 nd day, when a heavy admixture of pus cells appeared, which continued until slaughtering 2 months post inoculation. Mycoplasmas were isolated from the 7 th day; the number of viable organisms was as high as $10^{8}$ per ml. Post mortem: The left testis was enlarged, having a bulging cut surface from which a clear fluid exuded. In the caput epididymidis the clinically observed swelling was found to be of fibrotic nature; besides this lesion 3 small pus-filled foci were found in the corpus and a small haemorrhage in the cauda epididymidis. The left ampulla was enlarged, $11 \mathrm{~mm}$ in diameter, compared to $9 \mathrm{~mm}$ for the right one. Microscopic examination of the left testis revealed a few lymphocytic foci with very few eosinophils. The caput epididymidis was the site of a fibrotic inflammation, the corpus of an eosinophilic abscess formation with accumulation of eosinophils in the canal. The lesions in the left ampulla were of a more acute character with epithelial hyperplasia and extensive accumulations of eosinophilic leucocytes in the interstitia and lumina, together with many plasma cells. Mycoplasmas could be recovered from the left testis, epididymis, and ampulla and from the prepuce.

Bull " $K$ ". Clinical examination revealed that the left epididymis and vesicular gland were absent. The right vesicular gland was of normal size but somewhat firm. During 2 months, until slaughtering, 10 ejaculates of varying quality were collected, usually with pus admixture. Mycoplasmas were recovered twice. Post mortem: The cut surface of the right vesicular gland was brownish and dry, and rather dry drops of secretion could be squeezed from the acini. Histological examination revealed lesions of the same character as the experimentally induced vesiculitis (Bull 89), with the characteristic eosinophilia. Mycoplasmas could be recovered from the prepuce only.

Conclusions. It is concluded that the strain (" $K$ ") of Mycoplasma is experimentally pathogenic for the genital tract of bulls, and it seems possible that the chronic vesiculitis diagnosed in 
the bull from which the strain originally was isolated was caused by mycoplasmas, since the lesions were of the same type as those produced by experimental infection.

Erik Blom and Henning Ernф

State Veterinary Serum Laboratory,

Copenhagen, Denmark.

\section{REFERENCES}

Blom, E. \& A. Dam: Seminal vesiculitis in the bull induced by local injection of E. coli. 18. World Vet. Congr., Paris 1967. In press.

Christensen, N. O.: Studies on the agglutinin formation in brucellar infection of the genitals of the bull. Acta path. microbiol. scand. 1948, 25, 202-209.

Ern $\varnothing, H .:$ Mycoplasmosis: Demonstration of pathogenicity of a Danish strain of mycoplasma. Acta vet. scand. 1967, 8, 184-185.

(Received May 5, 1967). 